\title{
The VenSpec suite on the ESA EnVision mission to Venus
}

J. Helbert, A. C. Vandaele, E. Marcq, S. Robert, C. Ryan, et al.

J. Helbert, A. C. Vandaele, E. Marcq, S. Robert, C. Ryan, G. Guignan, Y. Rosas-Ortiz, E. Neefs, I. R. Thomas, G. Arnold, G. Peter, T. Widemann, L. Lara, "The VenSpec suite on the ESA EnVision mission to Venus," Proc. SPIE 11128, Infrared Remote Sensing and Instrumentation XXVII, 1112804 (9 September 2019); doi: 10.1117/12.2529248

SPIE Event: SPIE Optical Engineering + Applications, 2019, San Diego, California, United States 


\title{
The VenSpec suite on the ESA EnVision mission to Venus
}

\author{
J. Helbert*a, A. C. Vandaele ${ }^{\mathrm{b}}$, E. Marcq ${ }^{\mathrm{c}}$, S. Robert ${ }^{\mathrm{b}}$, C. Ryan ${ }^{\mathrm{a}}$, G. Guignan ${ }^{\mathrm{c}}$, Y. Rosas-Ortiz ${ }^{\mathrm{a}}$, E.

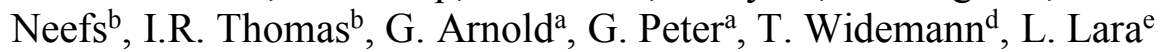 \\ ${ }^{a}$ Deutsches Zentrum für Luft- und Raumfahrt e.V. (Germany); ${ }^{b}$ Royal Belgian Institute for Space \\ Aeronomy, BIRA-IASB, Brussels, Belgium; 'LATMOS, Guyancourt, France; 'LESIA, Meudon,

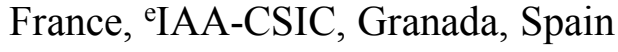

\begin{abstract}
The VenSpec instrument suite is part of the payload for the ESA M5 mission proposal EnVision which is currently in a competitive Phase A study.

VenSpec consists of three channels: VenSpec-M, VenSpec-H and VenSpec-U. VenSpec-M will provide near-global compositional data on rock types, weathering, and crustal evolution by mapping the Venus surface in five atmospheric windows. VenSpec-H will be dedicated to extremely high-resolution atmospheric measurements. The main objective of the VenSpec-H instrument is to detect and quantify $\mathrm{SO}_{2}, \mathrm{H}_{2} \mathrm{O}$ and $\mathrm{HDO}$ in the lower atmosphere, to enable characterization of volcanic plumes and other sources of gas exchange with the surface of Venus, complementing VenSAR and VenSpec$\mathrm{M}$ surface and SRS subsurface observations. VenSpec-U will monitor sulphured minor species (mainly $\mathrm{SO}$ and $\mathrm{SO}_{2}$ ) and the as yet unknown UV absorber in Venusian upper clouds and just above.

In combination, VenSpec will provide unprecedented insights into the current state of Venus and its past evolution. VenSpec will perform a comprehensive search for volcanic activity by targeting atmospheric signatures, thermal signatures and compositional signatures, as well as a global map of surface composition
\end{abstract}

Keywords: Venus, new mission, surface \& atmosphere composition, instrumentation.

\section{INTRODUCTION}

The VenSpec instrument suite on the EnVision mission [1] consists of three channels: VenSpec-M, VenSpec-H, VenSpec-U, and the Central Control Unit (CCU).

VenSpec-M will provide near-global compositional data on rock types, weathering, and crustal evolution by mapping the Venus surface in five atmospheric windows. The broadest window at $1.02 \mu \mathrm{m}$ is mapped with two filters to obtain information on the shape of the window. Additional filters are used to remove clouds, water, and stray light.

VenSpec-M will use the methodology pioneered by VIRTIS on Venus Express but with more and wider spectral bands, the VenSAR-derived DEM, and EnVision's circular orbit to deliver near-global multichannel spectroscopy with wider spectral coverage and an order of magnitude improvement in sensitivity. It will obtain repeated imagery of surface thermal emission, constraining current rates of volcanic activity following earlier observations from Venus Express.

VenSpec-H will be dedicated to high resolution atmospheric measurements. The main objective of the VenSpec$\mathrm{H}$ instrument is to detect and quantify $\mathrm{SO}_{2}, \mathrm{H}_{2} \mathrm{O}$ and $\mathrm{HDO}$ in both the troposphere and the mesosphere, to enable characterization of volcanic plumes and other sources of gas exchange with the surface of Venus, complementing VenSAR and VenSpec-M surface, SRS subsurface observations and VenSpec-U observations in the upper cloud layer. A nadir pointed high-resolution infrared spectrometer is the ideal instrument for these observations in different spectral windows between 1 and 2.5 microns, that permit measurements of the troposphere during the night, and of the mesosphere during the day. VenSpec-U will monitor sulphured minor species (mainly $\mathrm{SO}$ and $\mathrm{SO}_{2}$ ) and the as yet unknown $\mathrm{UV}$ absorber in Venusian upper clouds and just above. It will therefore complement the two other channels by investigating how the upper atmosphere interacts with the lower atmosphere, and especially characterize to which extent outgassing processes such as volcanic plumes are able to disturb the atmosphere through the thick Venusian clouds. A twin channel (0.2 nm in high-

Infrared Remote Sensing and Instrumentation XXVII, edited by Marija Strojnik, Gabriele E. Arnold, Proc. of SPIE Vol. 11128, $1112804 \cdot$ ? 2019 SPIE · CCC code: 0277-786X/19/\$18 · doi: 10.1117/12.2529248 
resolution, $2 \mathrm{~nm}$ in low-resolution) spectral imager in the $190-380 \mathrm{~nm}$ range able to operate in nadir would be especially suited to such a task.

In combination, VenSpec will provide unprecedented insights into the current state of Venus and its past evolution. VenSpec will perform a comprehensive search for volcanic activity by targeting atmospheric signatures, thermal signatures and compositional signatures, as well as a global map of surface composition.

\section{THE VENSPEC SUITE}

\subsection{VenSpec Consortium}

The VenSpec consortium is shown in Figure 1 which also shows the responsibilities of each partner with respect to the VenSpec channels. The goal of the consortium structure is to leverage the synergies between the three instruments and minimize the resources, while at the same time ensuring that each partner can develop their contributions largely independent. The current structure is a good balance between a fully integrated instrument and three completely independent instruments and builds on a long history of collaboration between the consortium partners.

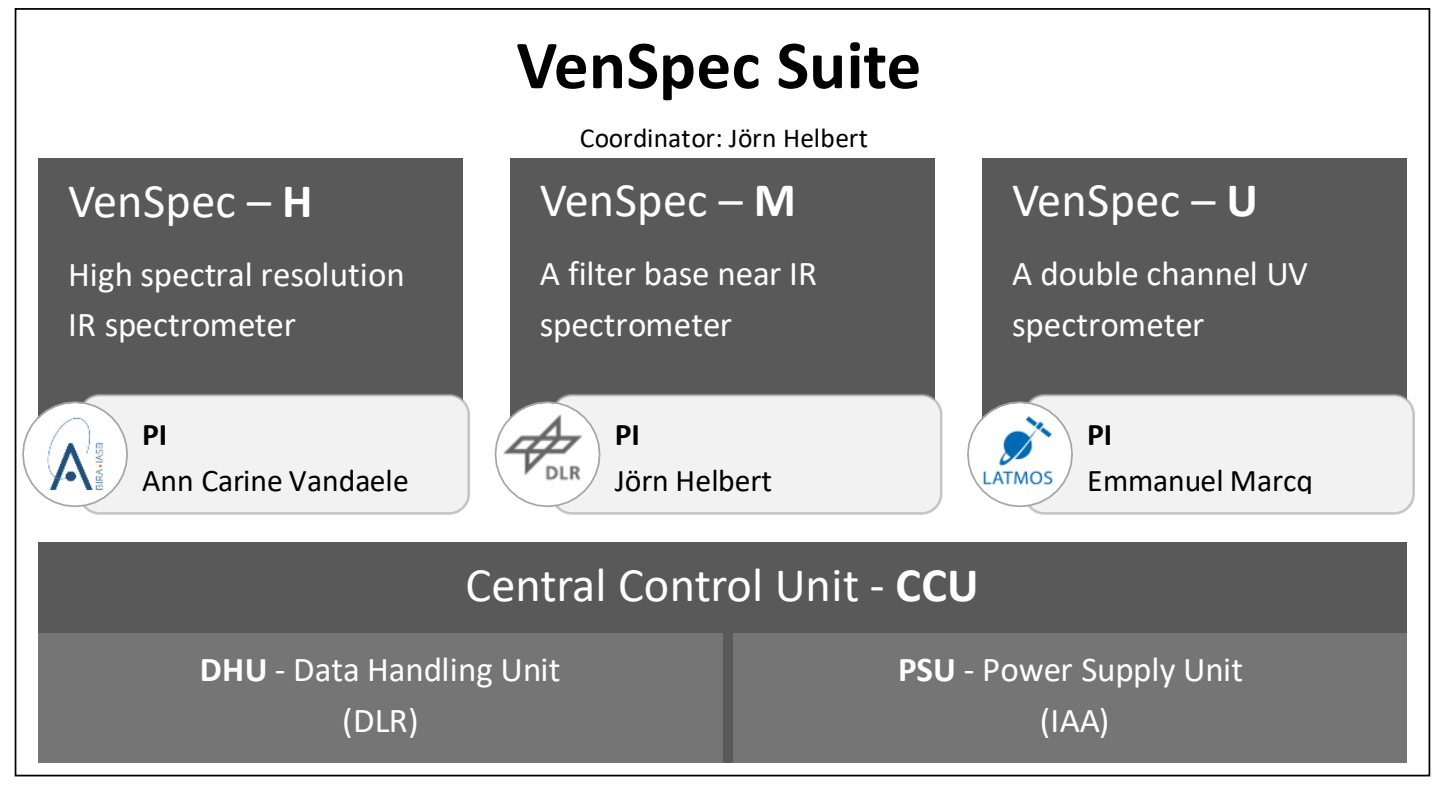

Figure 1 The VenSpec consortium

\subsection{VenSpec Suite}

The VenSpec suite consists of the three channels and a Central Control Unit (CCU). The concept is show in Figure 2. All three channels have their independent optics due to the very different imaging concepts and wavelengths ranges covered. The instruments are all nadir pointing. VenSpec-H requires Sun observations for calibration purposes whether these are obtained with a dedicated Sun port as shown in Figure 2 or via a pointing mirror is part of the Phase A study.

Each instrument has its own electronic unit for functionalities dedicated to that instrument. The central control unit contains two sub-units, the Data Handling Unit (DHU) and the Power Handling and Distribution Unit (PHDU). The DHU will handle the data interface to the spacecraft and can perform common control functions for all units. The PHDU will provide dedicated voltages to each of the instruments in the VenSpec suite. The details of the functionality of the CCU will be part of the Phase A study.

Thermally VenSpec will require a stabilized temperature reference point (e.g. a coldfinger) for the optics and detectors of VenSpec-M and VenSpec-U. For VenSpec-H a dedicated radiator might be required pending a detailed performance analysis in Phase A. 


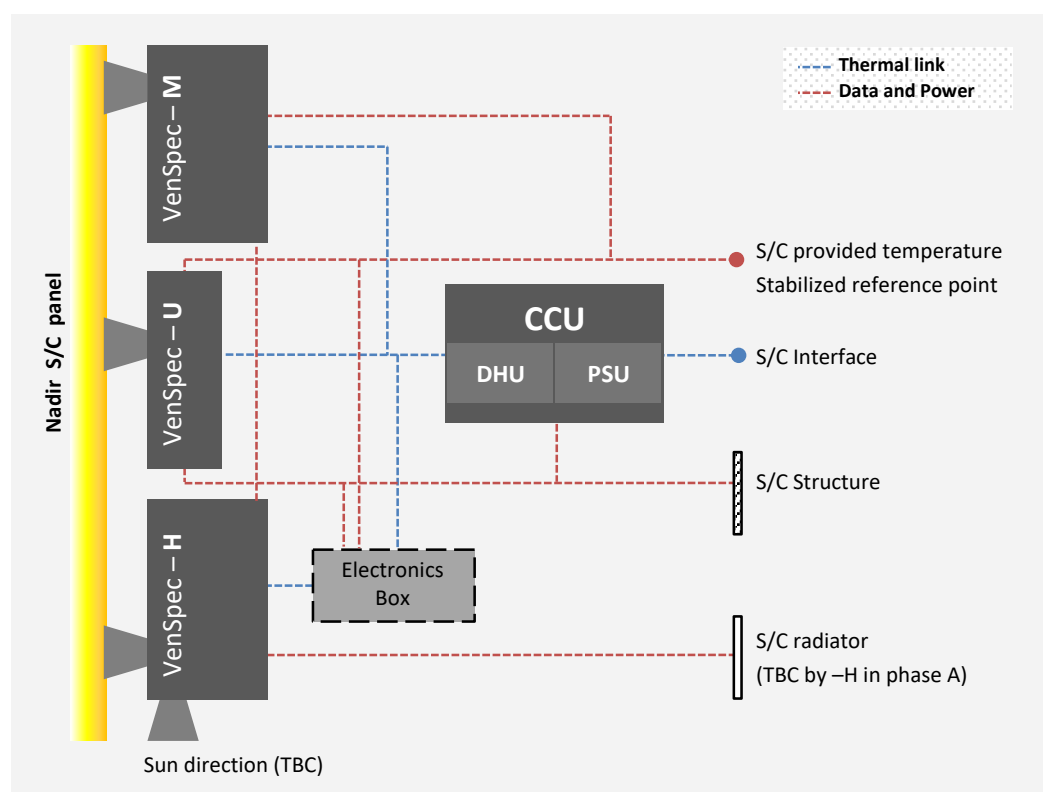

Figure 2 Block diagram of the VenSpec instrument suite

\subsection{VenSpec-H}

VenSpec-H is a high-resolution infrared spectrometer working in nadir viewing mode. It is an echelle grating spectrometer coupled to a high-performance, actively-cooled SOFRADIR HgCdTe detector, with an AcoustoOptic Tunable Filter (AOTF) at its entrance for order selection (Figure 3). These components are optimised for Venus atmospheric observations in the spectral range 1.0-2.5 $\mu \mathrm{m}$. This instrument has direct heritage from NOMAD-LNO [2, 3] which is currently onboard ExoMars Trace Gas Orbiter.

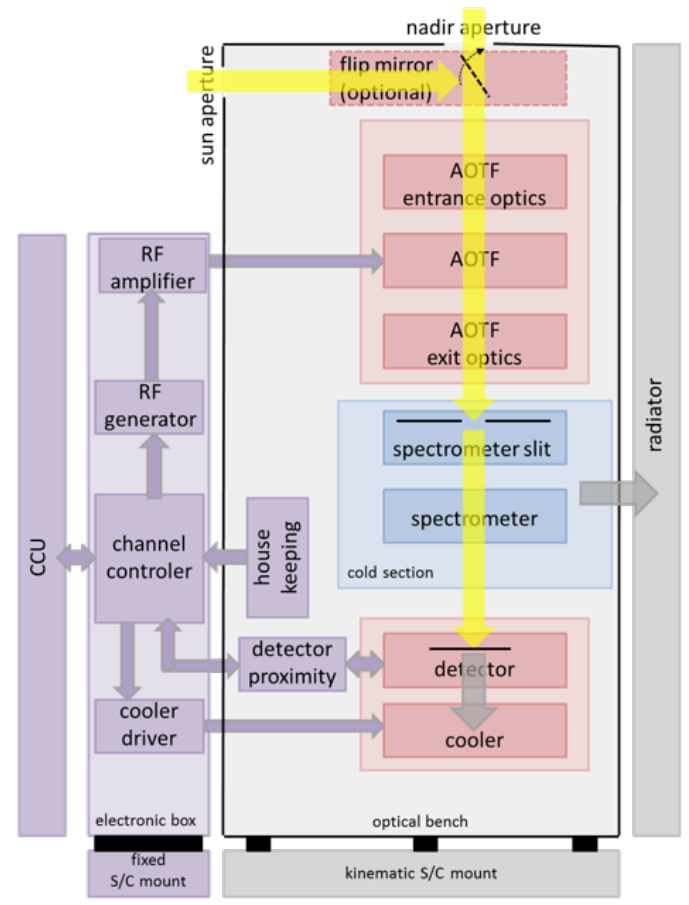

Figure 3: Block diagram of the VenSpec-H instrument 
The optical path of NOMAD-LNO (Figure 4) is reused for VenSpec-H. The occultation capability (periscope and flip mirror) is skipped in the baseline version of VenSpec-H since it is a pure nadir oriented instrument. However, calibration of the instrument might require pointing to the Sun. If this cannot be provided by s/c manoeuvres, the flip mirror mechanism might be needed to be implemented again (see Figure 3). The optics of the VenSpec-H channel are divided in three main units (Figure 5):

- Entrance optics that collects the light, defines the FOV and restricts the observed wavelength domain using an AOTF;

- Spectrometer with an echelle grating that defines the free spectral range and the instrument line profile (ILP); and

- Detector system that records the spectra.

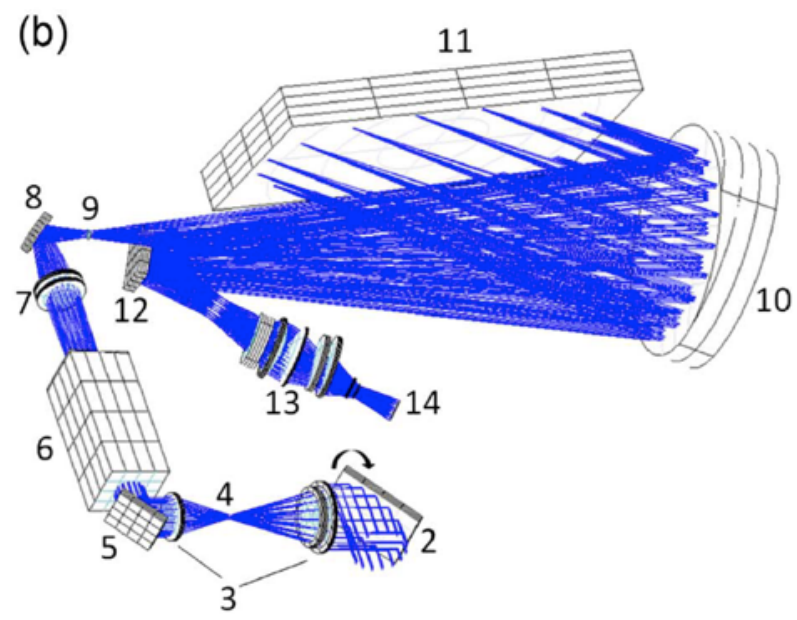

Figure 4: VenSpec-H optical path: (2) entrance mirror; (3-4-5) AOTF entrance optics; (6) AOTF; (7-8) AOTF exit optics; (9) spectrometer entrance slit; (10) parabolic mirror; (11) grating; (13) detector optics; (14) detector-cooler

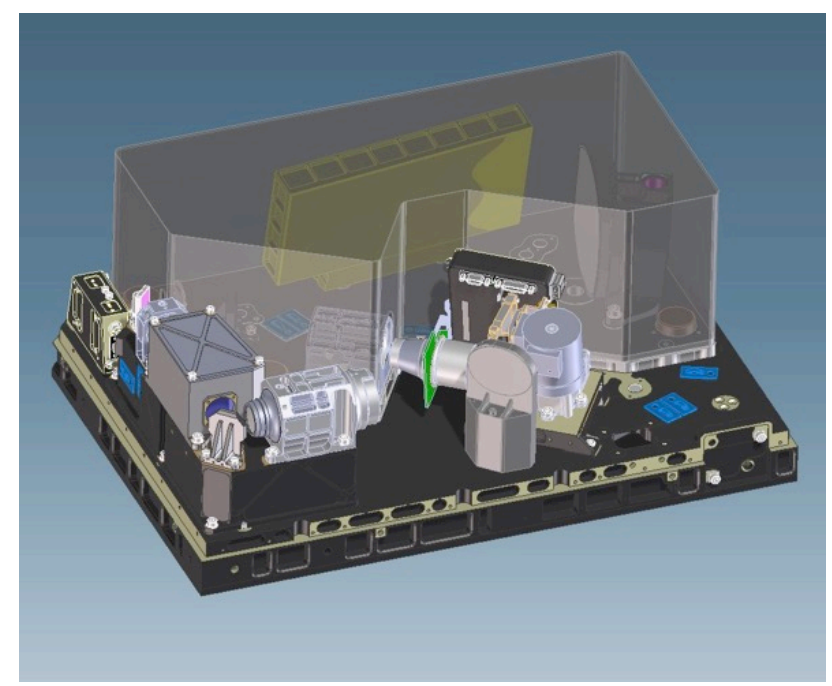

Figure 5: Optical Bench with cover over the cold section 
The optical path will be adapted:

- at its entrance, to fit the EnVision mission conditions;

- at its output, to match to a new detector (SOFRADIR Neptune instead of Mars);

- $\quad$ and overall, to fit to the available instrument volume.

To compact the design, the spectrometer is used in a quasi-Littrow configuration. The collimating and imaging lenses are merged in one off-axis parabolic mirror. The fast response of the AOTF allows quasi-simultaneous measurements of interesting atmospheric constituents to be performed, through almost instantaneous access into any wavelength domain within the AOTF's frequency parameters.

The echelle grating will be manufactured by Advanced Mechanical and Optical Systems (AMOS) Ltd in Belgium. The grating will cover all wavelength orders between 1.0 and $2.5 \mu \mathrm{m}$ and provide the requested resolution and spectral sampling.

The detector is a slightly modified standard Integrated Detector Dewar Cooler Assembly (IDDCA, type Neptune, see Figure 6) from SOFRADIR, France. It contains a high-sensitivity Focal Plane Array (FPA) of $30 \times 30 \mu \mathrm{m}$ $\mathrm{HgCdTe} / \mathrm{MCT}$ photovoltaic cells arranged in 500 columns (spectral direction) and 256 rows (spatial direction). The alloy mixing ratio is optimised for the 1.0 to $2.5 \mu \mathrm{m}$ spectral range of the science requirements at an FPA temperature of $85 \mathrm{~K}$. It is mounted in an evacuated Dewar with a customised optical window and cooled by a K508 closed cycle miniature Stirling cooling machine from RICOR (Israel), adapted for space applications.
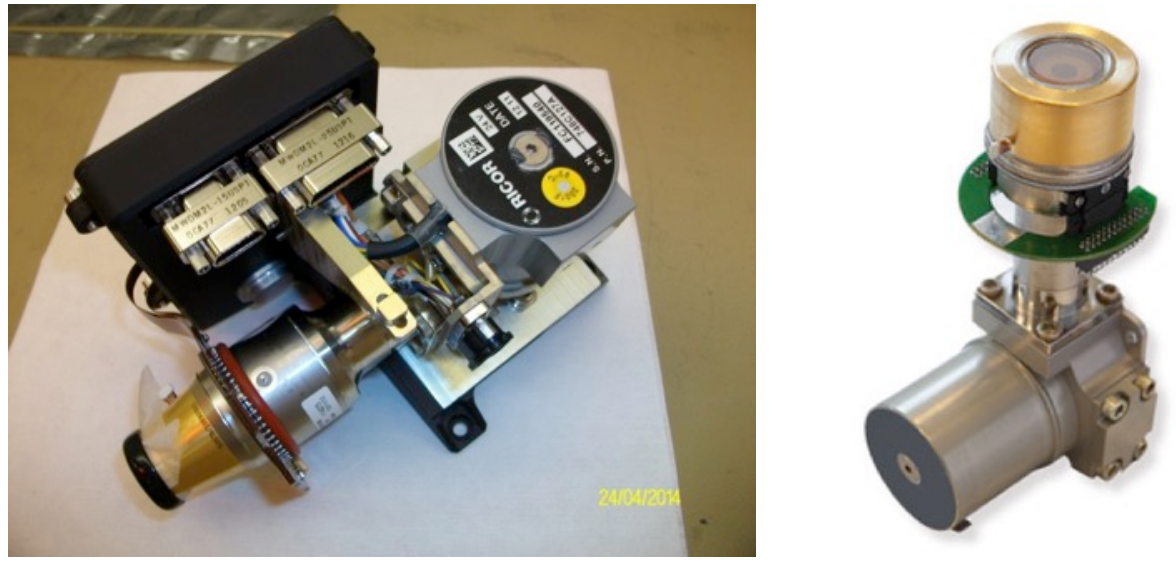

Figure 6: NOMAD-LNO detector/cooler assembly (left) and VenSpec-H detector/cooler assembly (right)

Static optical elements, such as parabolic mirror and grating, will be similar to the NOMAD-LNO ones. Only tweaking of their characteristics (e.g. line density and blaze angle of the grating) as function of the new optical parameters will be needed. 


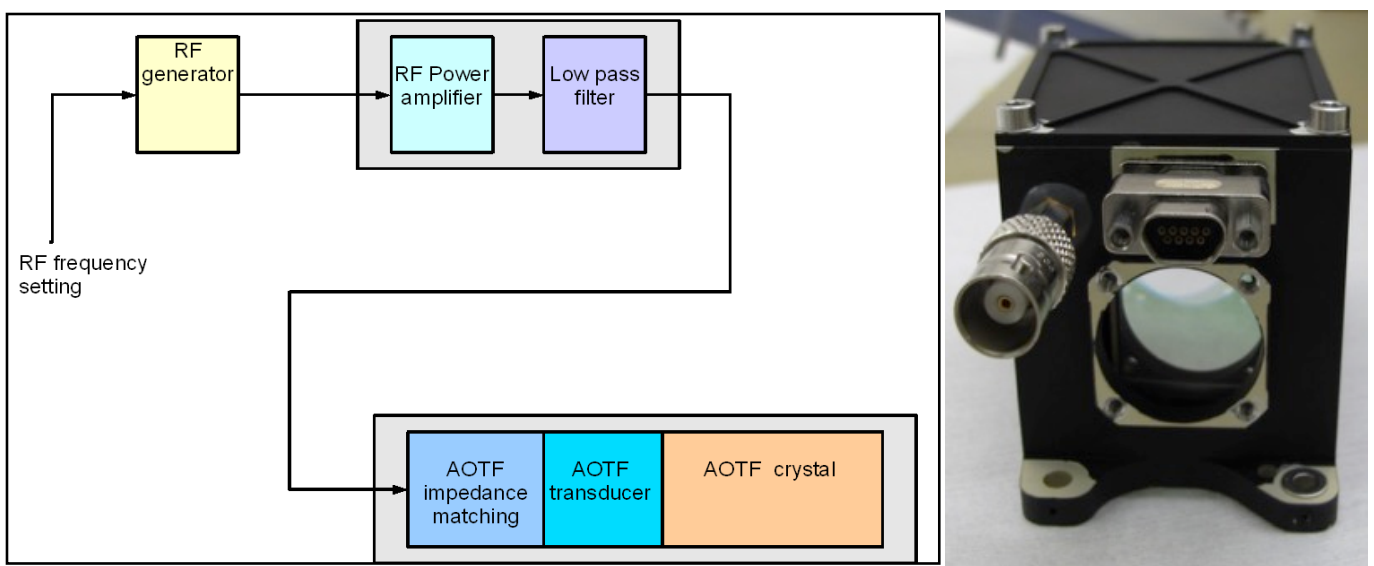

Figure 7: AOTF concept and picture

It will also be investigated whether an AOTF is needed as order sorter at the entrance of the instrument or if a simpler approach is possible, depending on the science needs (Figure 7). If an AOTF is used, it will be adapted to the spectral range of the VenSpec-H instrument.

Some electronic modules of VenSpec-H will be identical to the ones of NOMAD-LNO. Some others will need to be redesigned. E.g. the cooler driver electronics module can be reused (same cooler) while the detector proximity electronics will have to be redesigned (different FPA).

The RF Generator (based on Direct Digital Synthesis) and RF Amplifier are both heritage from NOMAD-LNO and can be reused, although alternative solutions with heritage exist (ALTIUS RF Generator based on analog Phase Locked Loop approach).

The NOMAD-LNO channel was controlled by an FPGA. This component is obsolete now and shall be replaced by a state-of-the-art (TBD) FPGA. The central control and housekeeping boards will therefore need a redesign. This includes also a redesign of the firmware and on-board software.

It is expected that a regulated $28 \mathrm{~V}$ is delivered to VenSpec-H. If not, heritage power supply solutions can be explored (e.g. Low Power Low Cost DC/DC converter from Thales-Belgium).

An Electrical Ground Support Equipment (EGSE) was developed for NOMAD. A similar EGSE will be built for VenSpec-H.

NOMAD-LNO was mounted in a standard upright position on top of another NOMAD channel. For VenSpec$\mathrm{H}$ the mechanical structure will be redesigned with the optical bench mounted upside down at the bottom of the base plate. The top of the base plate will serve as radiator to cool passively (part of) the optical path (cold section). The base plate is fixed to the $\mathrm{S} / \mathrm{C}$ with kinematic mounts. The main electronics block is separated from the optical path and fixed directly to the $\mathrm{S} / \mathrm{C}$ so that its internal heat production is not interfering with the optics (Figure 8).

The acceptable operational temperature range is $-30^{\circ} \mathrm{C}$ to $+40^{\circ} \mathrm{C}$ and the non-operational range is $-40^{\circ} \mathrm{C}$ to $+50^{\circ} \mathrm{C}$. A preliminary SNR investigation showed that the temperature of the (cold) spectrometer section should be $-50^{\circ} \mathrm{C}$ to $-30^{\circ} \mathrm{C}$ to reach the desired SNR. The detector temperature (active cooling) has to be $150 \mathrm{~K}$. However this temperature values will be investigated in detail during Phase A.

The VenSpec-H electronics is self-contained, allowing mounting on the deck of the spacecraft itself, avoiding additional thermal input into the optical bench of the instrument. 


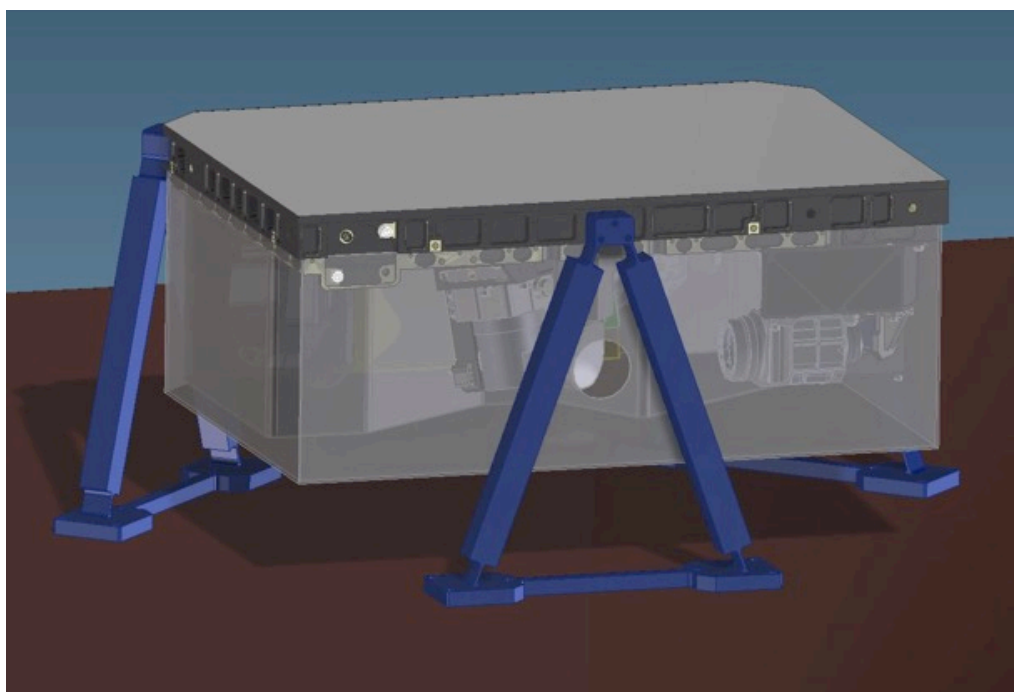

Figure 8: VenSpec-H mechanical concept

\subsection{VenSpec-M}

VenSpec-M consists of three units mounted together in a mono-block structure to allow for simplified SC interfaces: The optics and detector unit, the baffle functionally dedicated to the optics including a transparent aperture cover, and the control unit, including PCB's for internal power conditioning and instrument control and internal harness. The electronics box is the mechanical base of the instrument, providing interfaces to the optics and the baffle

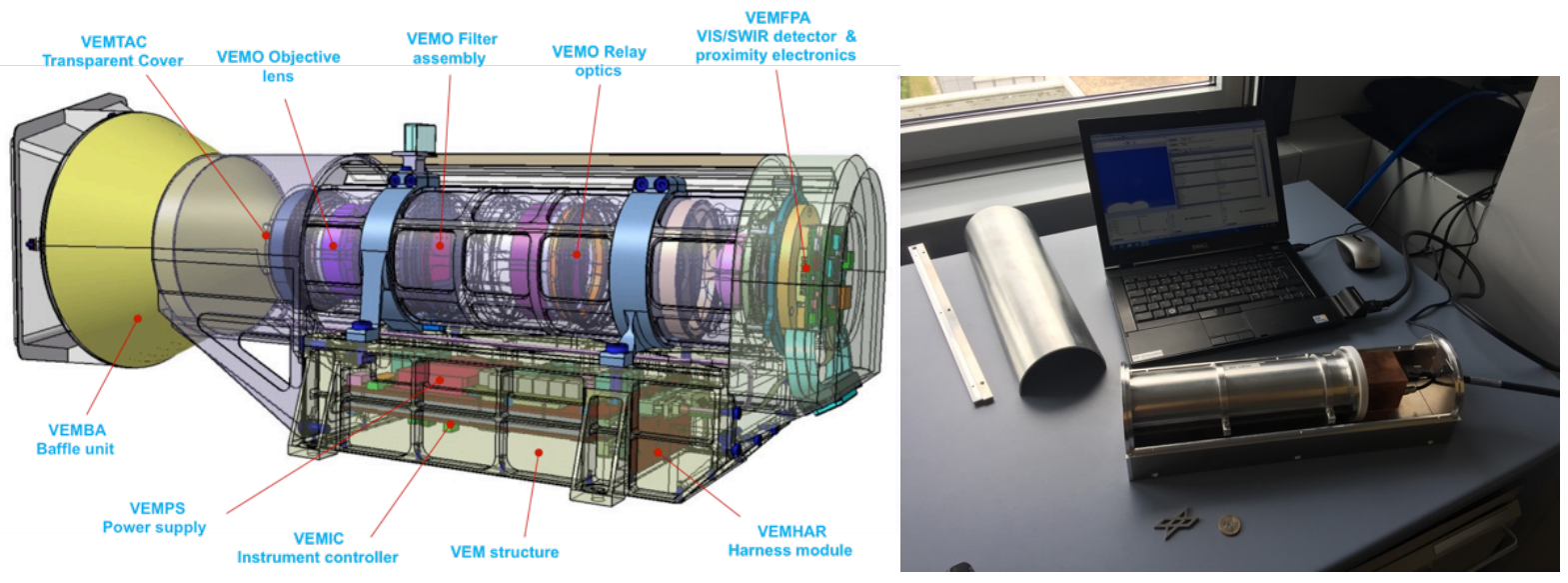

Figure 9 left: Current VenSpec-M design, right: VenSpec-M prototype representing upper part of the instrument with flight like optics and detector (top cover removed), instrument controller is replaced by a laptop

The VenSpec-M system design was discussed in detailed in as the Venus Emissivity Mapper for the NASA Discovery mission VERITAS [4, 5], so only a top-level summary is provided here. VenSpec-M is a pushbroom multispectral imaging system. The telecentric optics images the scene onto a filter array, and the image is relayed by a three-lenses objective onto the detector. The optical sub-system sits on top of the electronics compartment, which includes the Instrument Controller and the power supply (Figure 10). A two-stage baffle protects VenSpec-M from scattered light. A $45^{\circ}$ FOV yields a swath width of $207 \mathrm{~km}$ at an altitude of $250 \mathrm{~km}$, providing a thorough sampling of surface emissivity and orbit-orbit repeat coverage. 


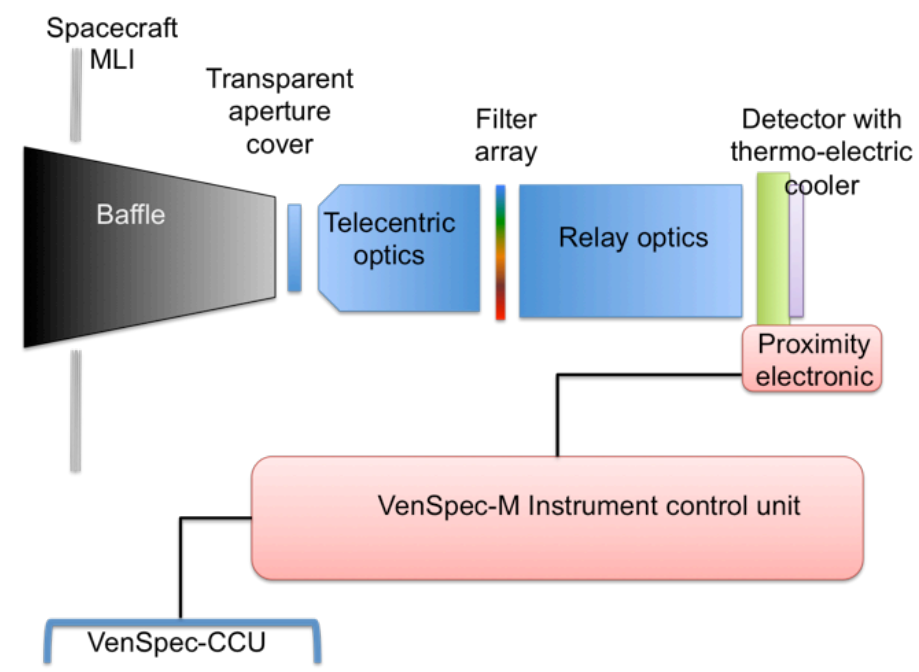

Figure 10 VenSpec-M design concept

VenSpec-M uses a thermal design developed for the MERTIS instrument on BepiColombo[6-8]. One thermal reference point (TRP) is the instrument mounting plate, which handles the electronics thermal control in an appropriate operating range with heater maintenance as needed. The optics/detector section is connected via a thermal strap to a stabilized spacecraft interface, which is the second TRP. The optics mounting elements thermally decouple both units. These facilities keeping the optics and detector stabilized in a very narrow temperature of $20^{\circ} \mathrm{C}+/-2^{\circ} \mathrm{C}$ while the electronics unit can accommodate a much wider temperature range. A thermal electric cooler integrated into the detector keeps the detector temperature stabilized to better than $100 \mathrm{mK}$. The baffle is thermally linked to the base unit and thermally isolated from the optics. Therefore the heatload on the baffle can be dissipated via the spacecraft structure without affecting the optics unit.

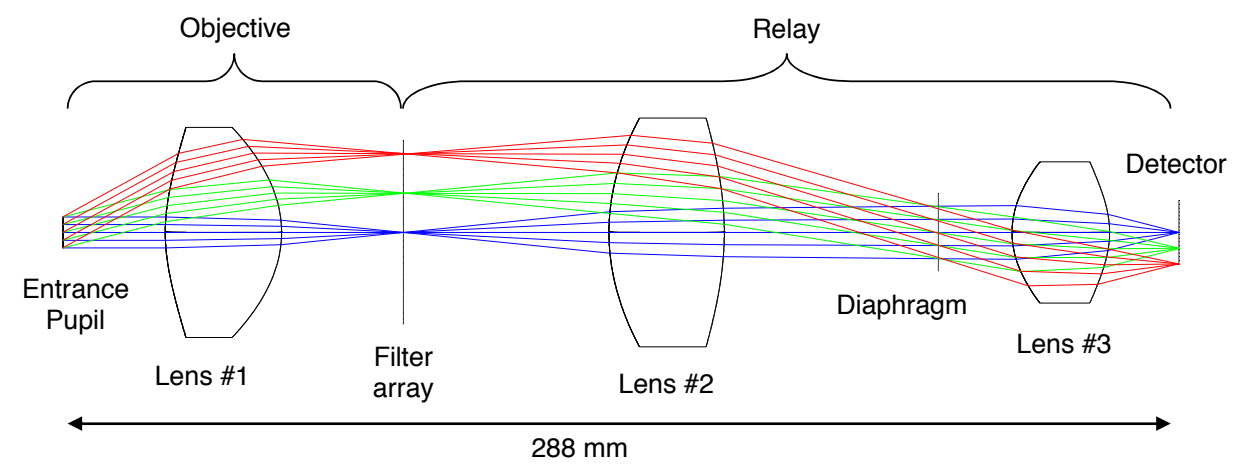

Figure 11 VenSpec-M optics design

The VenSpec-M optics (Figure 11, Table 1) has a field of view (FOV) of $45^{\circ}$, equivalent to $307 \mathrm{~km}$ from the nominal orbit altitude. Each pixel resolves $0.07^{\circ} \times 0.07^{\circ}(303 \mathrm{~m})$, which with an integration time of $90 \mathrm{~ms}$, leads to pixel dimension of $303 \mathrm{~m}$ across and $1000 \mathrm{~m}$ along track. Each ultra-narrow-band filter (made by Materion) occupies 33 of the 640 pixels across track; these are binned along and across track into $10 \times 10 \mathrm{~km}$ cells at the top of the clouds (for cloud correction) and $60 \times 60 \mathrm{~km}$ cells at the surface, providing a SNR of at least 300 for the cloud correction band at $<1.5 \mu \mathrm{m}$ and $>500$ for the mineralogical bands. This approach provides contiguous spectral emissivity coverage with the $10 \mathrm{~km}$ orbit advance. 
Table 1 VenSpec-M optical design parameter

\begin{tabular}{|l|l|l|}
\hline Parameter & Value & Comment \\
\hline Wavelength Range & $0.78-1.55 \mu \mathrm{m}$ & \\
\hline Number of Filtering Stripes & 14 & \\
\hline Total Track & $288 \mathrm{~mm}$ & From pupil to detector focal plane \\
\hline Instrument Focal Length & $16.4 \mathrm{~mm}$ & At detector level \\
\hline Instrument F/\# & 2.04 & At detector level \\
\hline Relay Magnification & $\times 0.4$ & \\
\hline Entrance Objective Focal Length & $40.5 \mathrm{~mm}$ & \\
\hline Entrance Objective F/\# & 5.06 & At filter array level \\
\hline Entrance Pupil Diameter & $8 \mathrm{~mm}$ & \\
\hline Field of View per Stripe & $45 \times 2.32 \mathrm{deg}$ & \\
\hline Detector Size & $640 \times 512 \mathrm{pixels}$ & $12.8 \times 10.24$ mm \\
\hline Pixel Pitch & $20 \mu \mathrm{m}$ & \\
\hline Pixel Angle & $0.070 \mathrm{deg}$ & \\
\hline Pixel Etendue & $7.5 \mathrm{e}-11 \mathrm{~mm}{ }^{2} . \mathrm{sr}$ & \\
\hline Filter Array Size & $32 \times 25.05 \mathrm{~mm}$ & Stripes 1.5 mm, Gaps 150 $\mu \mathrm{mm}$ \\
\hline Lens Shape & Aspheric & Only conic constant \\
\hline Material & K5G20 & RadHard Schott Glass \\
\hline Mass of the three lenses & $340 \mathrm{~g}$ & \\
\hline Total Lenses Transmission & 0.88 & 6 surfaces, 98\% transmission each \\
\hline
\end{tabular}

VenSpec-M minimizes the influence of stray light through two approaches. The first step is to physically reduce the amount of stray light entering the instrument aperture. VenSpec-M uses a two-stage baffle (Figure 12) with a light-weight front part that is mostly screening the $\mathrm{S} / \mathrm{C}$ but might get sun (aluminum, white ceramic coating, high temperature range) and a back part suppressing stray-light (3D selective laser melting of $\mathrm{TiAl}_{6} \mathrm{~V}_{4}$ - multiple black vanes, outside gold). The inner baffle is a multi-vane structure. Its baffle design exploits the arrangement as well as the number of the vanes, resulting in multiple internal reflection of out-of-field light. The absorptive coating supports the function of the baffle. For the first iteration of the baffle, 12 vanes were used to enhance the effectiveness of the baffle independent of the applied coating. That effect could be seen on the uncoated baffle manufactured during the Phase A study for the NASA Discovery VERITAS proposal (Figure 12). The electrolytic oxide Kepla-coat surface processing results in a degree of absorption greater than $95 \%$. The combination of the multi-vane structure and absorptive coating provides a significant margin to the required absorption of $80 \%$.

The second step to minimize stray light is to remove residual stray during data processing using dedicated filter channels. A full stray light analysis and a stray light test with a fully assembled and coated baffle are planned for a Phase B of the VenSpec development as part of the functional performance test on the engineering prototype. 


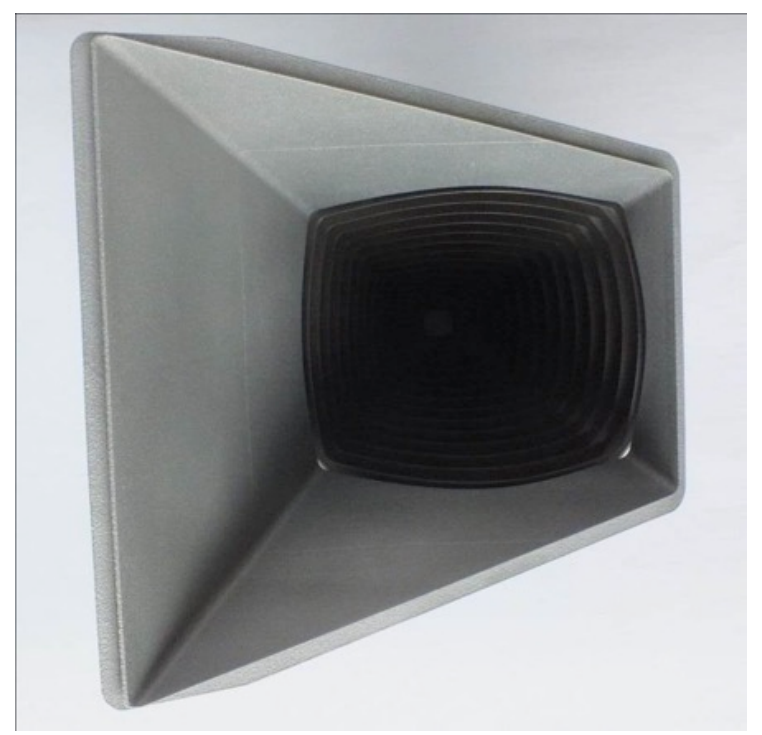

Figure 12 View into the baffle prototype, pre-manufactured without any absorbing or reflective coatings, shows the efficiency of the multi-vane design.

An optional transparent aperture cover (using fused silica protects the optics in cruise. Observations can be performed through the window. In the case of contamination on the window, it may be opened using a springloaded one-shot mechanism.

VenSpec-M obtains continuous night-side nadir observations in all spectral bands. To disentangle the surface and atmospheric contributions to the observed radiances, VenSpec-M uses an improved version of the extensively tested data pipeline developed to process VIRTIS surface data. The pipeline performs radiometric and geometric corrections and projections, corrects the radiance of surface windows for cloud opacity, and retrieves surface emissivity using topography obtained in parallel by VenSAR.

DLR developed the photometric, geometric, and atmospheric corrections for the VIRTIS surface data pipeline and plan to integrate radiative transfer methods during the Phase A study. It is planned to study in phase A to use for all VenSpec channels a MERTIS-derived instrument controller and power supply within a common housing but the different optical requirements need separate apertures and optics to maximise performance with only a marginal mass penalty.

\subsection{VenSpec-U}

VenSpec-U is a dual channel (low resolution, hereafter LR: 190-380 nm; and high resolution, hereafter HR: 190-240 nm) UV spectral imager designed to monitor cloud top abundances of volcanic sulphured gases (SO, SO2) as well as UV contrasts through spectral analysis of backscattered sunlight on the day side of Venus. The block diagram of the instrument is presented in Figure 13.

Each channel consists of:

- An entrance baffle, used to limit the straylight coming from the Venus disk

- An objective composed of 2 lenses and a stop diaphragm

- A spectrometer mainly composed of a slit and a toroidal holographic grating; it also comprises a low pass filter to reject the wavelengths above the higher limit of the bands ( $>240 \mathrm{~nm}$ for $\mathrm{HR},>380 \mathrm{~nm}$ for LR) and a zero-order trap (to avoid straylight due to internal reflexions of the zero-order of the gratings - TBC)

Both slits (LR and HR) are parallel and the optical layout is such that both channels have the same instantaneous field of view (IFOV) allowing simultaneous observation of the same target as well as simultaneous 
calibration on the same star. Each slit image is then spectrally dispersed by its respective toroidal holographic grating and is formed on a shared CMOS back-side illuminated (BSI) detector. Therefore, the narrow-slit axis of the detector contains the spectral information, whereas the long-slit axis contains the spatial information along the $22.5^{\circ}$ FOV of each slit. The spectra of LR and HR channels are dispersed one above the other on the focal plane.

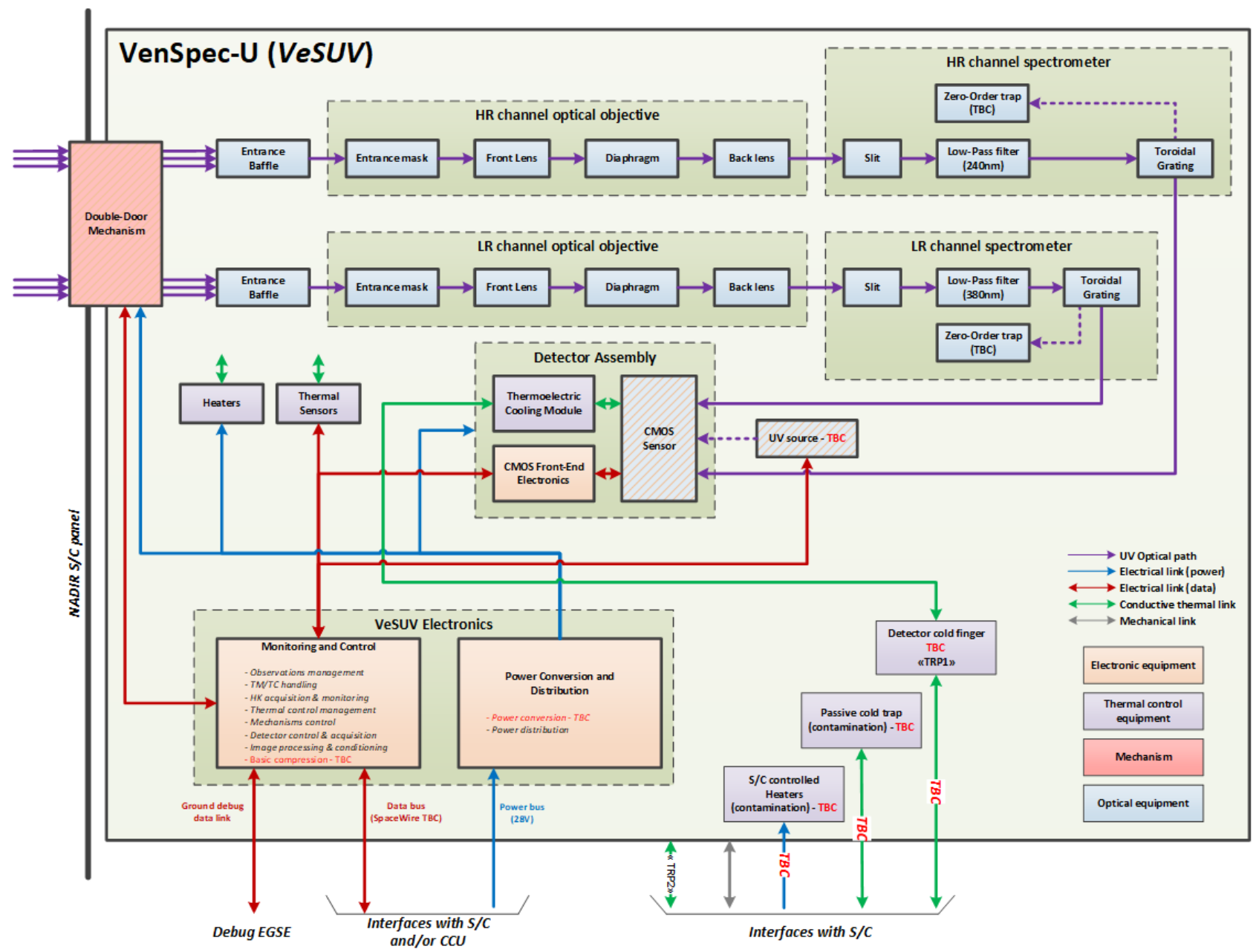

Figure 13 Block diagram of VenSpec-U

The detector package includes a TEC element used to cool down and regulate the detector allowing low dark current and stable behaviour.

Binning on the spatial axis is performed on the detector, enabling a SNR $>100$ for HR and $>200$ for LR (resulting in a coarser spatial resolution on the more dispersed HR channel). The remaining spatial direction is provided through orbital scrolling, an observational strategy known as pushbroom. Observations can be conducted in a strict nadir geometry (null emission angle), or in near-nadir geometry. The detector will be controlled such that the integration time and the binning scheme is adjusted independently (and simultaneously) for each channel giving high flexibility and parameters optimisation for each acquisition (SNR vs. spatial resolution vs. altitude).

The instrument optical entrance is protected against high fluxes and contamination by a door mechanism (that will be open only during observations and closed the rest of time). This "light-tight" door will be also used for dark measurements allowing mid/long-term dark current monitoring (ageing, radiation). The type and position of the door are TBD (upstream or downstream the baffle). The general optical layout contains two separate entrance optics per channel (Figure 14). The use of a small UV source to perform in-flight flatfield correction of the detector is under study. As an UV instrument, VenSpec-U is sensitive to contamination. In addition to the 
adoption of a "cleanliness-oriented design", several items (TBC) may be required to limit the degradation of the performances to an acceptable level:

- On ground: sintered filters (protection during AIT and venting during launch), $\mathrm{N}_{2}$ purging interface(s)

- In-flight: heaters, for optics decontamination purpose, and Zeolite pellets, for molecules trapping

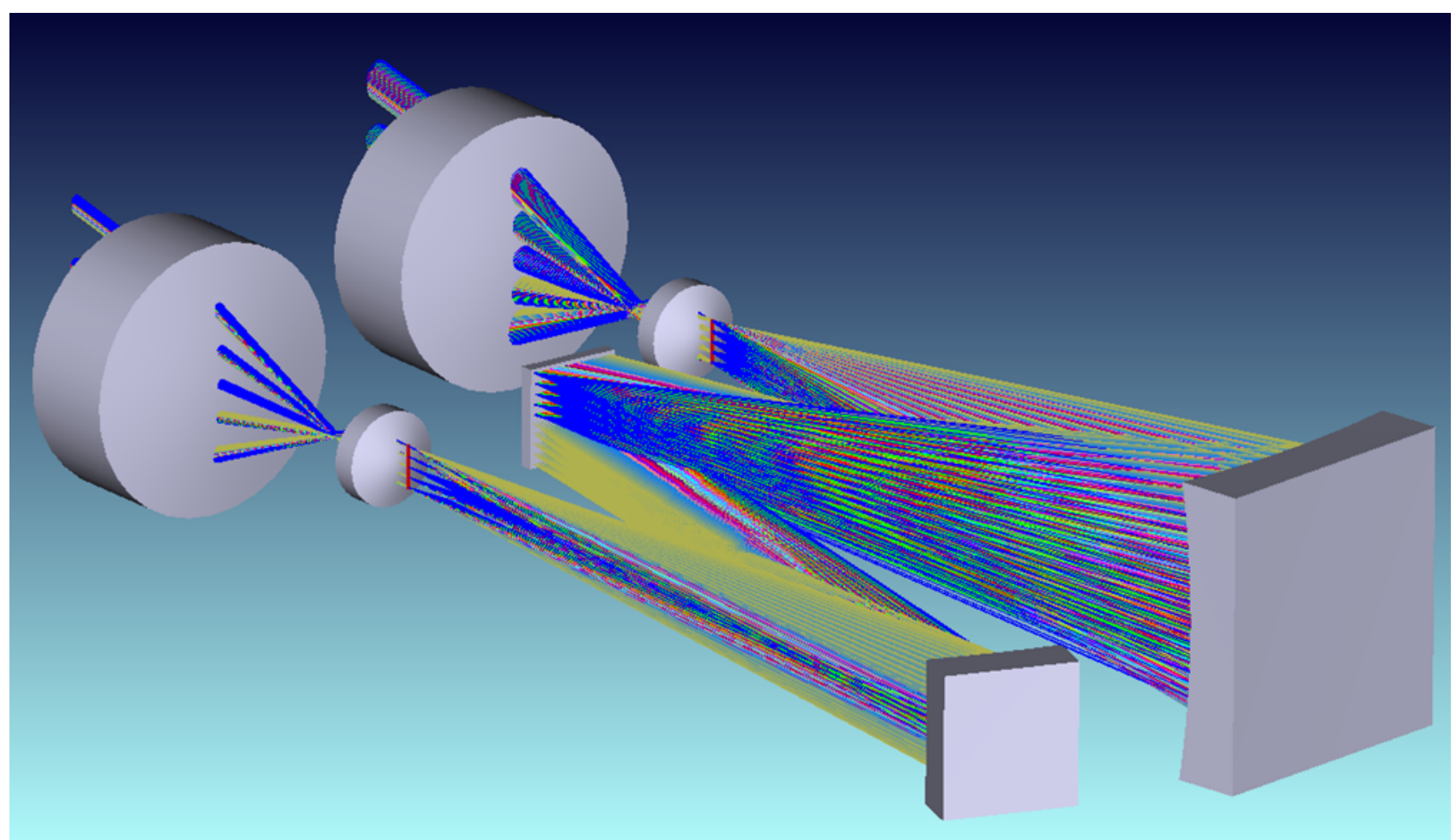

Figure 14 VenSpec-U optical scheme

A summary of the optical layout is presented in Table 2.

Table 2 Optical data for VenSpec-U

\begin{tabular}{|l|l|l|l|}
\hline & LR channel & HR channel & Comments \\
\hline Wavelength range [nm] & $190-380$ & $190-240$ & \\
\hline Spectral resolution (FWHM, nm) & 2 & 0.2 & \\
\hline Binned spatial sampling [km²] & $3 \times 3$ & $12 \times 12$ & $\begin{array}{l}\text { LR: SNR }>200 \\
\text { HR: SNR }>100\end{array}$ \\
\hline Slit dimensions & $\begin{array}{l}9 \mathrm{~mm} \times 180 \mu \mathrm{m} \\
\text { TBC }\end{array}$ & $\begin{array}{l}9 \mathrm{~mm} \times 70 \mu \mathrm{m} \\
\mathrm{TBC}\end{array}$ & \\
\hline Slit Field of View (FOV, height of the slit) & $22.5^{\circ}$ & $22.5^{\circ}$ & \\
\hline Slit spatial magnification & $\sim 1$ & $\sim 1$ & \\
\hline Optical scheme dimensions & $\begin{array}{l}\text { Envelope in } \\
300 x 200 x 100\end{array}$ & the order of & $\begin{array}{l}\text { Without baffles. Envelope includes : } \\
\text { Objectives, spectrometers, detector }\end{array}$ \\
\hline
\end{tabular}




\begin{tabular}{|c|c|c|c|}
\hline & LR channel & HR channel & Comments \\
\hline Gratings & \multicolumn{2}{|c|}{$\begin{array}{l}\text { Aberration Corrected, Toroidal } \\
\text { Holographic }\end{array}$} & \\
\hline \multirow[t]{2}{*}{ Groove density [grooves/mm] } & 514.691 & 1945.067 & \\
\hline & $\mathrm{TBC}$ & $\mathrm{TBC}$ & \\
\hline Dimensions $\left[\mathrm{mm}^{2}\right]$ & $\sim 65 \times 65$ & $\sim 35 \times 35$ & \\
\hline Curvature radii $[\mathrm{mm}]$ & $\sim 200 \mathrm{TBC}$ & $\sim 200 \mathrm{TBC}$ & \\
\hline Entrance objective & \multicolumn{2}{|c|}{$\begin{array}{l}2 \text { aspheric lenses, UV grade } \\
\text { fused Silica }\end{array}$} & \\
\hline Focal length & $23 \mathrm{~mm}$ & $23 \mathrm{~mm}$ & \\
\hline F-\# & $\sim 4,3 \mathrm{TBC}$ & $\sim 11 \mathrm{TBC}$ & \\
\hline Front lens diameter & $60 \mathrm{~mm}$ & $60 \mathrm{~mm}$ & \\
\hline Low pass filters & \multicolumn{2}{|c|}{ UV grade fused silica } & \\
\hline Dimensions $\left[\mathrm{mm}^{2}\right]$ & $12 \times 23 \mathrm{TBC}$ & $6 \times 15$ TBC & \\
\hline Cutoff wavelength & $\sim 380 \mathrm{~nm}$ & $\sim 240 \mathrm{~nm}$ & \\
\hline Detector & \multicolumn{2}{|l|}{ Common } & \\
\hline Useful area $\left[\mathrm{mm}^{2}\right]$ & \multicolumn{2}{|c|}{$20($ spatial $) \times 20($ spectral $)$} & \\
\hline Pixel size & \multicolumn{2}{|c|}{$10 \mu \mathrm{m} \times 10 \mu \mathrm{m}$ TBC } & \\
\hline Full well charge & \multicolumn{2}{|l|}{$45 \mathrm{ke}^{-} \mathrm{TBC}$} & With $10 \mu \mathrm{m} \times 10 \mu \mathrm{m}$ pixels \\
\hline Readout noise & \multicolumn{2}{|c|}{ 4e- to $11 \mathrm{e}-\mathrm{RMS}$ TBC } & (single pixel, no binning) \\
\hline Power [W] & \multicolumn{2}{|l|}{4} & \\
\hline
\end{tabular}

\subsection{VenSpec-CCU}

The Central Control Unit (CCU) consists of two sub-units, the Data Handling Unit (DHU) and the Power Supply Unit (PSU). It will be developed under the overall leadership of DLR.

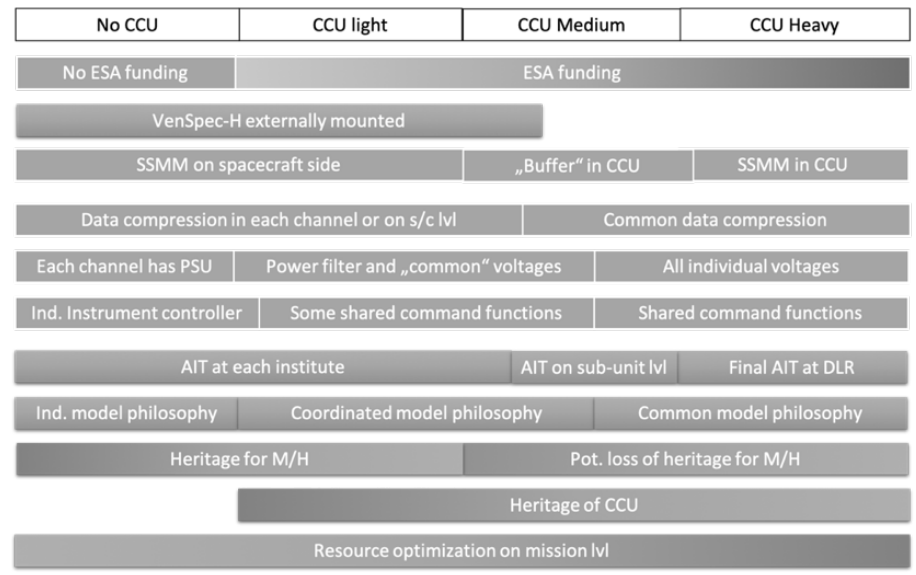

Figure 15 Starting point for the CCU complexity trade-off 
The DHU will incorporate heritage from a wide range of missions for which DLR has provided DHUs, including Rosetta, VenusExpress, PLATO, CHEOPS and JUICE. At this stage the MERTIS instrument controller (ICU) and power supply is used as a baseline for the development of the DHU; which consists of one ACTEL FPGA including LEON-FT processor core, 128 Mbyte SDRAM and 2 MByte EEPROM. The PSU development will be handled by IAA in Spain, based on their extensive with power supplies for BepiColombo, Rosetta, JUICE and PLATO. The design of the PSU will be derived from PHU designs for the JUICE GALA and BepiColombo BELA instrument.

In order to maximize the benefits of the VenSpec CCU early in Phase A, a trade study needs to be performed between complexity of CCU and independence of instruments for integration, testing and calibration, see Figure 15 .

\section{OUTLOOK}

EnVision is currently in a competitive Phase A study, with a selection expected in summer of 2021. The work in the Phase A study focuses on consolidating the design for an integrated VenSpec suite with a Central Control Unit. In coordination with ESA and the industrial primes studying the spacecraft implementation a number of trade-off studies will be performed to optimize the configurations.

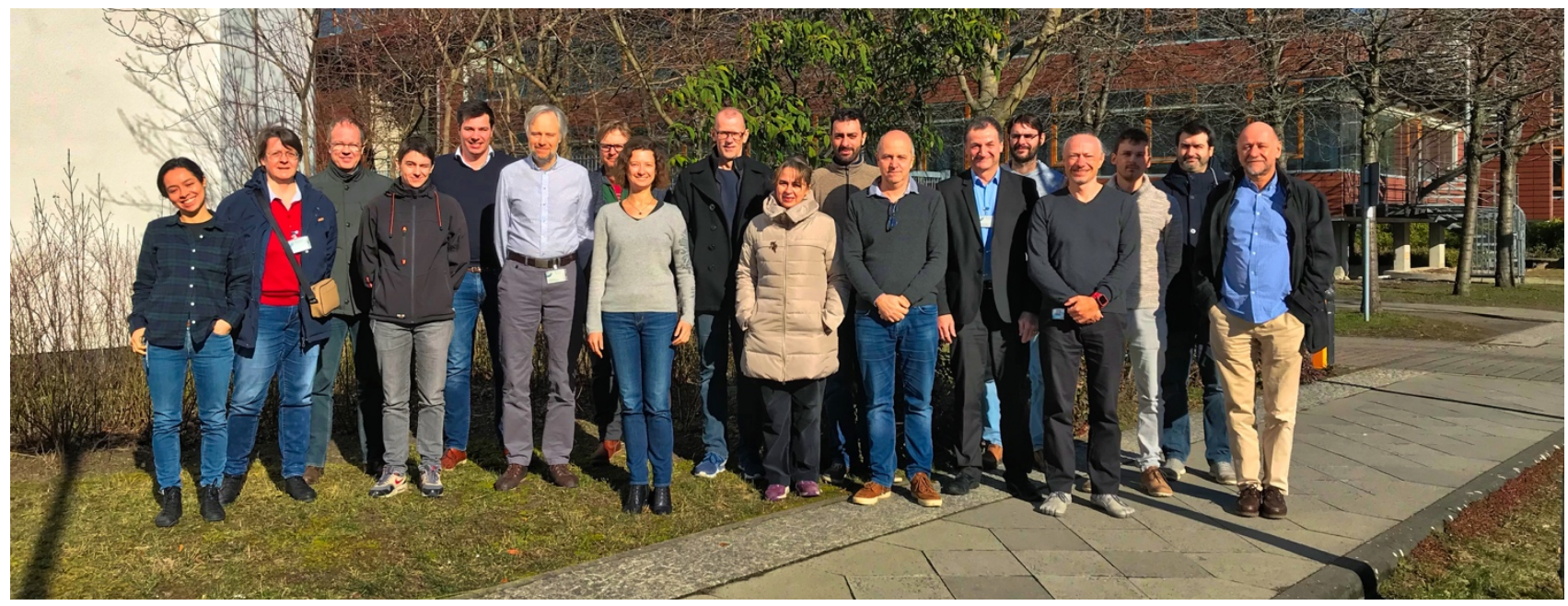

Figure 16 VenSpec consortium and ESA study team at the Phase A kickoff meeting

The VenSpec suite will provide unique insights into the evolution of Venus by providing measurements covering the whole range from the surface to the top of the atmosphere.

\section{REFERENCES}

[1] R. C. Ghail, C. Wilson, M. Galand et al., "EnVision: taking the pulse of our twin planet," Experimental Astronomy, 33(2-3), 337-363 (2012).

[2] E. Neefs, A. C. Vandaele, R. Drummond et al., "NOMAD spectrometer on the ExoMars trace gas orbiter mission: part 1--design, manufacturing and testing of the infrared channels," Appl Opt, 54(28), 8494-520 (2015). 
[3] A. C. Vandaele, J. J. Lopez-Moreno, M. R. Patel et al., "NOMAD, an Integrated Suite of Three Spectrometers for the ExoMars Trace Gas Mission: Technical Description, Science Objectives and Expected Performance," Space Science Reviews, 214(5), (2018).

[4] J. Helbert, D. Wendler, I. Walter et al., "The Venus Emissivity Mapper (VEM) concept," Proceedings of SPIE. 9973, 99730R-99730R-13.

[5] J. Helbert, M. D. Dyar, I. Walter et al., [The Venus Emissivity Mapper (VEM): Obtaining global mineralogy of Venus from orbit], (2018).

[6] I. Walter, G. Paez, T. Zeh et al., "Deep space instrument design for thermal infrared imaging with MERTIS," 8154, 81540Y (2011).

[7] H. Hiesinger, and J. Helbert, "The Mercury Radiometer and Thermal Infrared Spectrometer (MERTIS) for the BepiColombo mission," Planetary and Space Science, 58(1-2), 144-165 (2010).

[8] J. Helbert, H. Hiesinger, I. Walter et al., "MERTIS: understanding Mercury's surface composition from mid-infrared spectroscopy," 7808, 78080J (2010). 